\title{
Radio Frequency Ablation of right sided accessory pathway - 8 years experience at SGNHC
}

\author{
Raut R, KC MB, Rajbhandari S, Dhungana M, Shah R, Shah KB
}

Shahid Gangalal National Heart Centre (SGNHC)

Address for Correspondence:

Dr. Roshan Raut

Tel: 00977-1-4371322, 00977-1-4371374

Fax: 00977-1-4371123

E-mail: rautroshan@hotmail.com

\begin{abstract}
Background

Radiofrequency ablation has been established as the curative treatment for most of supraventricular tachycardia since 1987. In SGNHC, ablation has been done routinely since 2003. In our experience, right sided accessory pathway (AP) has been technically difficult and challenging with low success rate as mentioned in literature outside. So, the objective of this article was to analyze the demographic characteristics, success and recurrence rate and complication of right sided AP ablation in our centre.

Methods and Materials

From $13^{\text {th }}$ Oct 2003 to $9^{\text {th }}$ Sept. 2011, altogether 768 patients who underwent ablation were analyzed.

Result

Among 768 patients, 416(54.2\%) were accessory pathways out of which 72(17.3\%) were right sided AP. Majority of right sided accessory pathway were manifest (WPW) comprising $77.1 \%$, significantly higher compare to left sided pathway where manifest AP was only $44.1 \%(p<0.001)$. Among all attempted ablation of right sided AP, 11 ablations failed so that the success rate was $84.7 \%$ lower than that of left sided pathway where success rate was $98.4 \%(p<0.001)$. Among 61 successful ablations, 2 relapsed during follow up period, recurrence rate being 3.3\%. Although there were few complications in the ablation of other tachyarrhythmia, there was no complication noted during the ablation of 72 right sided AP.
\end{abstract}

\section{Conclusion}

Right sided pathway are mostly manifest. The success rate of right sided pathway is lower in comparison to left sided pathway. Our success rate is comparable to the result in the literature which indicates that ablation of right sided accessory pathway in our centre is reasonably good.

\section{Key Words}

Right sided accessory pathway, Radiofrequency ablation 


\section{Introduction}

The first radiofrequency catheter ablation of Supraventricular tachycardia in human was done in 1987 1. Since then, it has been established as the curative treatment for most of the supraventricular tachycardias ${ }^{2-4}$. Conventionally, according to the anatomic location, the accessory pathway is divided into left, septal and right sided pathway ${ }^{5}$ (Fig 1). Left and right sided pathways are further categorized as posterior, posterio-lateral, lateral, antero-lateral and anterior accessory pathway. Septal pathway is categorized as posterio-septal, mid-septal and antero-septal accessory pathway.

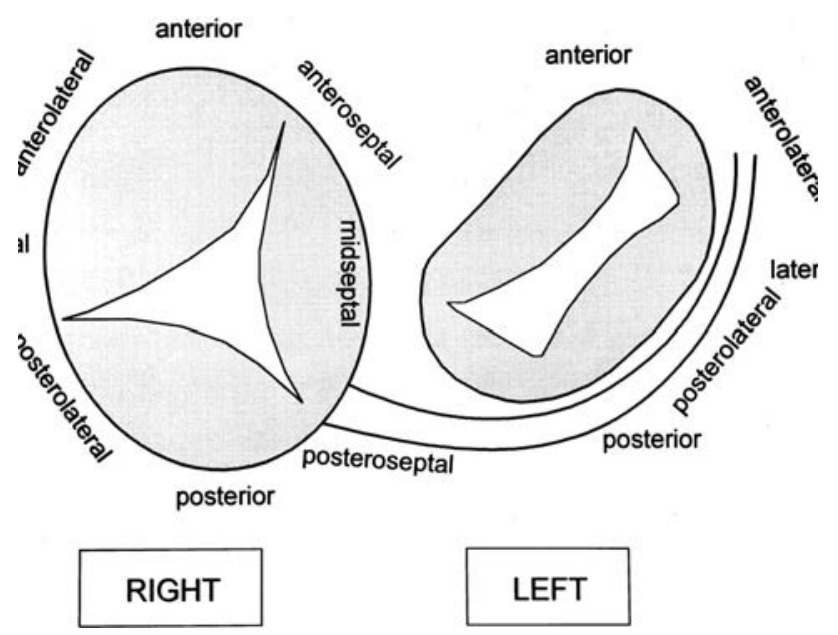

Fig 1. Localization of accessory pathway.

Literature shows that majority of right sided pathway have both antegrade and retrograde conduction so that right sided pathway mostly presents as manifest accessory pathway ${ }^{6}$. Apart from other factors, the probability of antegrade conduction also depend on the location of accessory pathway. In contrast to left sided AP, there is smaller interatrial distance for an impulse to conduct from sinus node to atrial input on the right sided AP so that there is greater chance to conduct antegradely via right sided AP 6. Compare to left sided pathway, right sided pathway is technically difficult to ablate and is associated with lower success and higher recurrence rate ${ }^{7,8}$.

The reason behind this are ${ }^{9}$ :

1. Important anatomic differences exist between the tricuspid valve annulus and mitral valve annulus. The folding over the right atrial wall to right ventricle creates an acute angle of attachment of the tricuspid valve annulus resulting in less room available for manipulation of catheter tip.
2. Unstable catheter positioning in tricuspid annulus.

3. Absence of reference electrode in the right side like Coronary Sinus electrode in left side.

4. Higher incidence of congenital abnormality e.g. Ebsteins anamoly in tricuspid valve annulus.

It is thus the reported success rate in different studies of right sided pathway ablation are about $80-90 \%$ lower than that of left sided pathway. 7,8

The first ablation in our centre was done in $13^{\text {th }}$ Oct. 2003. Since then, we have done ablation for various type of tachyarrhythmia, mostly AVNRT and AVRT and few VT, atrial flutter, Mahaim and atrial flutter. In the past 8 years, we experienced that the right sided accessory pathway ablation was technically difficult and the success rate low. So, in this article, we reviewed the efficacy and demographic characteristics of right sided AP ablation.

\section{Objective}

The objective of this article was to evaluate the demographic characteristics and the efficacy of right sided AP ablation.

\section{Materials and methods}

Three $6 \mathrm{Fr}$ quadripolar catheters were placed in right atrium, His and right ventricle and one $6 \mathrm{Fr}$ decapolar catheter was placed in coronary sinus. The ablation of right sided pathway was done via right femoral venous approach. All the patients who underwent ablation from $13^{\text {th }}$ Oct 2003 to $9^{\text {th }}$ Sept. 2011 were reviewed in this article. The data regarding age, sex, manifest vs concealed, outcomes like successful ablation, attempted ablation, failure and relapse were extracted from the EPS data form. SPSS 16 was used for statistical analysis.

\section{Result}

Demographic Characteristics: All together 768 patients had undergone radiofrequency ablation. Among them, 322 (41\%) were AVNRT, 416 (54.2\%) were Accessory pathway and remaining 30 (3.9\%) were others including $\mathrm{VT}$, atrial flutter, Mahaim and PJRT. Among 416 APs, right sided pathway comprised 72 (17.3\%). The distribution of right sided AP in different location with success and recurrence is shown in table 1 . In right sided pathway, mean age was $33.4 \mathrm{yrs}$, male being $58.4 \%$. Although patients with AP has smaller age compared to AVNRT (35.9 vs 43.5 yrs, $\mathrm{p}<0.001$ ), there was no difference in age among different location of accessory pathways. 
Table 1. AP distribution on right side with success and recurrence rate.

\begin{tabular}{lccc|}
\hline Location & Number of AP (\%) & Success (\%) & Recurrence (\%) \\
\hline Right Anterior & $3(4.2 \%)$ & $2(66 \%)$ & 0 \\
\hline Right antero-lateral & $10(13.9 \%)$ & $8(80 \%)$ & 0 \\
\hline Right Free wall & $36(50 \%)$ & $31(86.1 \%)$ & $2(3.3 \%)$ \\
\hline Right Posterio-lateral & $15(20.8 \%)$ & $13(86.7 \%)$ & 0 \\
$\begin{array}{l}\text { Right posteri- } \\
\text { or }\end{array}$ & $8(11.1 \%)$ & $7(87.5 \%)$ & 0 \\
Total & 72 & $61(84.7 \%)$ & $2(3.3 \%)$
\end{tabular}

Concealed vs manifest: Majority of right sided accessory pathway were manifest (WPW) comprising $77.1 \%$. This no. was significantly higher compare to left sided pathway where manifest AP was only $44.1 \%(p<0.001)$.There was no significant difference according to the location of right sided AP.

Outcome: There was no unattempted ablation of right sided pathway. Among all attempted ablation, 11 ablations failed so that the success rate was $84.7 \%$ lower than that of left sided pathway where success rate was $98.4 \%(p<0.001)$. Although not statistically significant, it was observed that more anterior the right sided AP was, lower the success rate; anterior $66 \%<$ antero-lateral $80 \%<$ free wall $86.1 \%<$ posterior-lateral $86.7 \%<$ posterior $87.5 \%$. (Fig 2 )

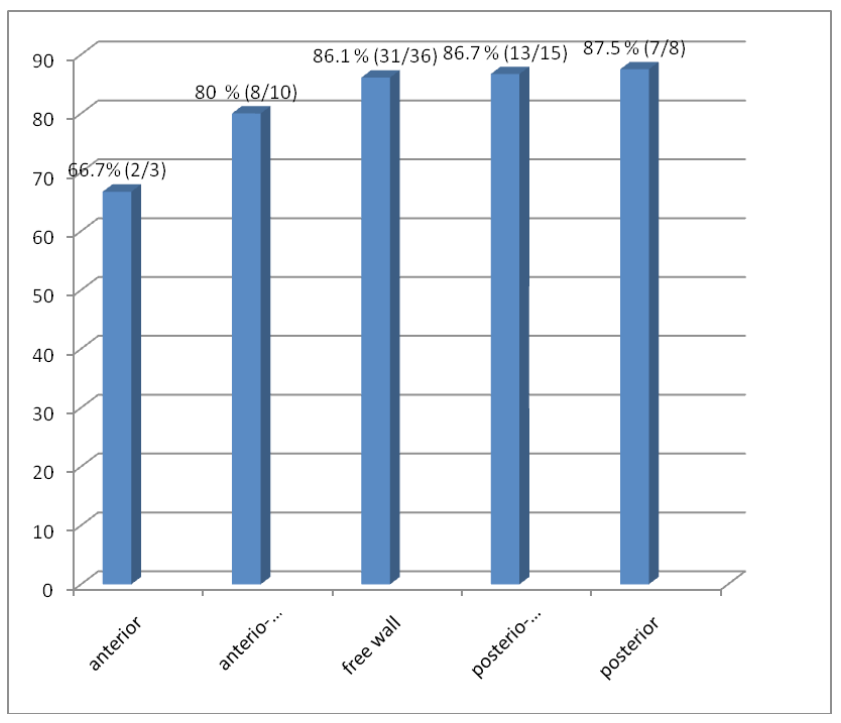

Fig 2. The success rate of right sided AP according to its location

Recurrence: Among 61 successful ablations, 2 relapsed during follow up period so that recurrence rate was only $3.3 \%$ much lower than that in the literature.

Complication: Although there were few complications in the ablation of other tachyarrhythmia, there was no complication noted during the ablation of 72 right sided AP.

\section{DISSCUSSION}

Majority of right sided pathway are manifest. As in the literature mentioned, it is due to the smaller distance for impulse to conduct from sinus node to right sided AP. Mean age is significantly lower compare to AVNRT. In our centre, the success rate was lower compare to left sided AP. We also found difficulty in mapping right sided AP as there was no reference electrode in right sided pathway like Coronary Sinus catheter in left sided pathway. Furthermore, the unstable ablation catheter made mapping and ablation more difficult. In our experience, more anterior the right sided AP was, more difficult to map and ablate and lower the success rate was. To maintain the catheter stability, we used long vascular sheaths like SR. Sometimes we used 7Fr ablation catheter for better support and stable positioning. Eventually, Our success rate was comparable to the various reported data in the literature. ${ }^{7,8}$ This clearly indicate that ablation of right sided pathway in our centre, inspite of being technically difficult and challenging, has quite acceptable result.

\section{CONCLUSION}

Right sided pathway are mostly manifest. The success rate of right sided pathway is lower in comparison to left sided pathway. Our success rate is comparable to the result in the literature which indicates that ablation of right sided accessory pathway in our centre is reasonably good. 


\section{Nepalese Heart Journal}

\section{References}

1. Huang SK, Bharati S, Graham AR, Lev M, Marcus Fl, Odell RC. Closedchest catheter dessication of the atrioventricular junction using radiofrequency energy: a new method of catheter ablation. J Am Coll Cardiol 1987; 9: 349-358.

2. Jackman WM, Wang X, Friday KJ, et al. Catheter ablation of accessory atrioventricular pathways (Wolff-Parkinson-White syndrome) by radiofrequency current. N Engl J Med 1991; 324: 1605-1611.

3. Calkins $H$, Sousa J, El-Atassi $R$, et al. Diagnosis and cure of the Wolff-Parkinson-White syndrome or paroxysmal supraventricular tachycardias during a single electrophysiologic test, N Eng J Med 1991,324:1612-1618.

4. Lesh MD, Van Hare GF, Schamp DJ, et al. Curative percutaneous catheter ablation using radiofrequency energy for accessory pathways in all locations: Results in 100 consecutive patients. J Am Coll Cardiol 1992,19:1303-1309.

5. Braunwald's Heart Disease. Seventh Edition.

6. Valentine Fuster, Richard A. Walsh et. al. Hurst's The Heart, $13^{\text {th }}$ edition.

7. Scheinman MM: Patterns of catheter ablation practice in the United States:Results of the 1992 NAPSE survey.North American Society of Pacing and Electrophysiology. Pacing Clin Electrophysiol 1994; 17:873.

8. Wong KT, Yung TC, Lun KS, et.al Ten-year Experience of Radiofrequency Catheter Ablation of Accessory Pathways in Children and Young Adults J Paediatr (new series) 2005; 10:257-264

9. Igor Singer. Interventional Electrophysiology, Second Edition. 